\title{
LKB1 inactivation occurs in a subset of esophageal adenocarcinomas and is sufficient to drive tumor cell proliferation
}

Timothy G. Whitsett, PhD, ${ }^{a}$ Sumeet K. Mittal, MD, ${ }^{a}$ Jennifer M. Eschbacher, MD, ${ }^{b}$ Vashti M. Carson, BS, Michael A. Smith, MD, ${ }^{\mathrm{a}}$ Ross M. Bremner, MD, PhD, ${ }^{\mathrm{a}}$ and Landon J. Inge, $\mathrm{PhD}^{\mathrm{a}}$

\section{ABSTRACT}

Background: The incidence of esophageal adenocarcinoma (EAC) has increased over the last several decades. Apart from mutations in TP53 gene, there are little data on genetic drivers of EAC. Liver kinase B1 (LKB1) has emerged as a multifunctional tumor suppressor regulating cell growth, differentiation, and metabolism. Somatic inactivation of $L K B 1$ has been described in several tumor types; however, whether LKB1 inactivation has a role in EAC is unknown. Here we analyzed patient tumors to assess the prevalence of LKB1 loss in EAC.

Methods: Chromosomal deletion and expression of $L K B 1$ in EAC were investigated using publicly available genomic data. Protein expression was assessed by immunohistochemistry (IHC) analysis for LKB1 in a tissue microarray (TMA) containing esophageal tumor specimens, including EAC. LKBI was suppressed in EAC cells to determine the effects on cell growth in vitro.

Results: Analysis of EAC data in The Cancer Genome Atlas dataset revealed significant deletion of chromosome 19p13.3, containing the $L K B 1$ gene locus. Single copy loss (shallow deletion) of $L K B 1$ was present in $58 \%$ of EAC samples. Expression of $L K B 1$ was significantly lower in EAC tumors compared with normal esophagus. IHC analysis showed reduced LKB1 protein expression in EAC. Suppression of $L K B 1$ was sufficient to enhance EAC cell growth in vitro.

Conclusions: Our data suggest that inactivation of LKB1 frequently occurs in EAC. Based on the reported oncogenic effects of LKB1 inactivation, our data indicate that $L K B 1$ loss may play a significant role in EAC tumorigenesis, and point to the need for future studies. (J Thorac Cardiovasc Surg 2018;155:1891-9)

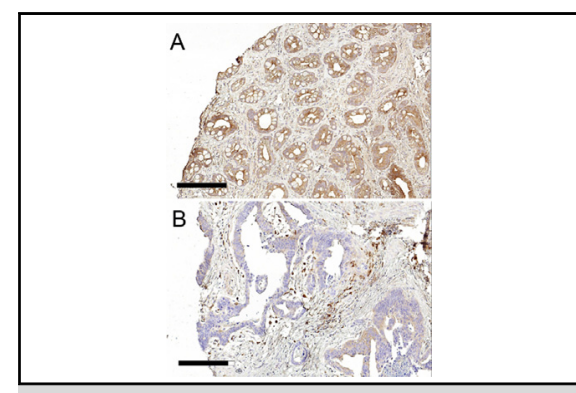

The tumor suppressor LKB1 is lost in a subset of EAC compared with intestinal metaplasia.

\section{Central Message}

Loss of liver kinase B1 (LKB1) is a common molecular event in esophageal adenocarcinoma (EAC). Because the inactivation of LKB1 is associated with poor prognosis, the role of LKB1 in EAC should be explored in this disease.

\section{Perspective}

The prevalence of esophageal adenocarcinoma (EAC) has increased over the past several decades. The molecular events that drive EAC progression are poorly understood. Our data suggest that $\mathrm{LKB} 1$ is lost in a subset of EAC tumors and is associated with earlier disease onset. Because loss of LKB1 is associated with tumor progression and poor prognosis, this tumor suppressor represents a potential driver of EAC.

See Editorial Commentary page 1900.

\footnotetext{
From the ${ }^{\mathrm{a} N o r t o n}$ Thoracic Institute, and ${ }^{\mathrm{b}}$ Department of Pathology, Barrow Neurological Institute, St Joseph's Hospital and Medical Center, Phoenix, Ariz. Funded by Norton Thoracic Institute Mission Support Funds (to TGW and LJI) Read at the 43rd Annual Meeting of the Western Thoracic Surgical Association, Colorado Springs, Colorado, June 21-24, 2017.

Received for publication June 21, 2017; revisions received Nov 8, 2017; accepted for publication Nov 16, 2017; available ahead of print Jan 19, 2018

Address for reprints: Landon J. Inge, PhD or Timothy G. Whitsett, PhD, Norton Thoracic Institute, St Joseph's Hospital and Medical Center, 124 W Thomas Rd, Suite 105, Phoenix, AZ 85013 (E-mail: Timothy.Whitsett@DignityHealth.org or Landon.Inge@DignityHealth.org). $0022-5223 / \$ 36.00$

Copyright (c) 2017 by The American Association for Thoracic Surgery https://doi.org/10.1016/j.jtcvs.2017.11.067
}

The incidence and mortality of esophageal cancer has seen a significant rise over the last few decades, with a concomitant shift in the predominant histological subtype in the United States from esophageal squamous cell carcinomas

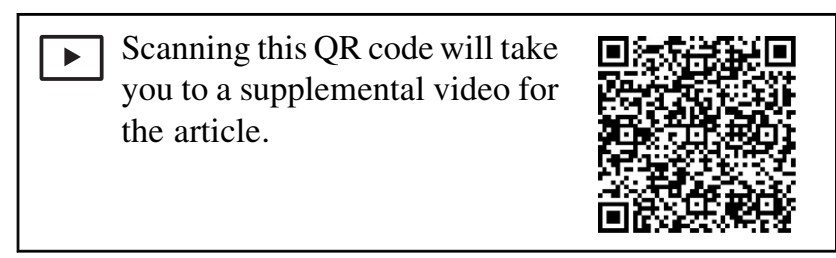




$$
\begin{aligned}
& \text { Abbreviations and Acronyms } \\
& \begin{aligned}
\text { BE } & =\text { Barrett's esophagus } \\
\text { EAC } & =\text { esophageal adenocarcinoma } \\
\text { FFPE } & =\text { formalin-fixed, paraffin-embedded } \\
\text { LKB1 } & =\text { liver kinase B1 } \\
\text { p-AMPK } & =\text { phosphorylated-protein kinase AMP- } \\
& \text { activated catalytic subunit alpha } 1 \\
\text { PJS } & =\text { Peutz-Jeghers syndrome } \\
\text { RPPA } & =\text { reverse-phase protein assay } \\
\text { STK11 } & =\text { serine/threonine kinase } 11 \\
\text { TMA } & =\text { tissue microarray } \\
\text { TCGA } & =\text { The Cancer Genome Atlas } \\
\text { TP53 } & =\text { tumor protein } 53
\end{aligned}
\end{aligned}
$$

(ESCCs) to esophageal adenocarcinomas (EACs). ${ }^{1}$ Esophageal cancer was expected to affect $\sim 17,000$ people in 2017 and has a dismal 5-year survival rate of only $18 \%{ }^{2}$ Current standard of care therapies for advanced EACs rely on chemotherapy and radiation, and are plagued by resistance and toxicity. ${ }^{3}$ This rise in incidence and the poor prognosis call for an investigation of the molecular events responsible for carcinogenesis and tumor progression, especially those that can dictate rational therapeutic strategies.

In 2013, Dulak and colleagues ${ }^{4}$ used exome sequencing to determine recurrent, molecular events in EAC. While validating the high prevalence of TP53 and CDKN2A tumor suppressor alterations, and demonstrating mutations in ELMO1 and DOCK2, their work did not identify many recurrent alterations in therapeutically targetable genes. Oncogenic amplifications were observed in receptor tyrosine kinases (RTKs), such as epidermal growth factor receptor (EGFR), Erb-B2 RTK 2 (ERBB2/HER2), or MET protooncogene RTK (MET), with actionable therapeutics occurring at later stages and following genome doubling events. ${ }^{5}$ Secrier and colleagues ${ }^{6}$ recently demonstrated amplification of multiple RTKs in single lesions, making targeted therapy more challenging. These complex, heterogeneous, and historically difficult-to-target molecular events (ie, loss of TP53 or CDKN2A) have hampered the introduction of novel therapeutic strategies against EACs. Thus, a deeper understanding of the molecular events that drive EAC is needed to provide guidance for therapeutic avenues.

Liver kinase B1 (LKB1), also known as serine/threonine kinase 11 (STK11), was discovered as a causal, genomic alteration in the hereditary cancer disorder known as Peutz-Jeghers syndrome (PJS). ${ }^{7} L K B 1$ has been further identified as a tumor-suppressor gene across a number of tumor types, with the highest prevalence of genomic alterations occurring in lung adenocarcinomas, ${ }^{8}$ affecting cell growth, invasion/metastasis, cellular metabolism/energetics, and immune infiltration. ${ }^{9}$ LKB1 loss of function can be accomplished via somatic mutations, genomic deletions, or epigenetic silencing. ${ }^{8,10}$ Although patients with PJS are more susceptible to gastrointestinal tract cancers, including cancers of the esophagus, ${ }^{11}$ little is known about the role or prevalence of LKB1 alterations in EAC. LKB1 expression levels, as determined by quantitative polymerase chain reaction or immunoblot analysis, were seen to be down-regulated in esophageal cancer compared with normal epithelium, ${ }^{12-14}$ although much of that work was done in ESCCs or used limited numbers of EACs. As such, the expression of LKB1 in EACs might inform prognosis and therapeutic decision making.

In the present study, we used a tissue microarray (TMA) with tumors from patients with EAC, as well as publicly available EAC genomic datasets, to better understand the expression of LKB1 in EAC. In these patients, LKB1 expression was correlated with clinical characteristics. Finally, we used knockdown of $L K B 1$ by siRNA in EAC cell lines to investigate tumor cell growth in vitro.

\section{METHODS \\ TMA Construction}

Archival esophageal specimens were obtained from deidentified patients who underwent surgical resection (ie, esophagectomy) between 2007 and 2010 at our institution under an Institutional Review Boardapproved study protocol (PHXA-17-0084-71-07; approved May 16, 2017). All tumor specimens were collected before initiation of therapy. Specimens chosen for the TMA after proper storage and handling in the hospital biobank were determined by a board-certified pathologist (JME) and verified for tumor content by hematoxylin and eosin (H\&E) staining. Here 2- $\mu \mathrm{M}$-thick, formalin-fixed, paraffin-embedded (FFPE) cores of tumor, high-grade dysplasia, or Barrett's esophagus (BE) were selected by pathological review of H\&E sections and arranged in a single FFPE block using the Arraymold Kit A tissue microarray system (Arraymold, Riverton, Utah). Multiple cores (2-4) of each patient sample were used to construct the TMA.

\section{Immunohistochemistry}

Immunohistochemistry (IHC) analysis of LKB1 was performed as described previously. ${ }^{15}$ In brief, $5-\mu \mathrm{M}$ sections of the EAC TMA were adhered to charged microscope slides. Baking, deparaffinization, and heat-induced epitope retrieval and IHC staining were performed on the Leica BOND RXm autostainer (Buffalo Grove, Ill). Antibodies to LKB1 (clone D60C5F10) and phosphorylated protein kinase AMP-activated catalytic subunit alpha 1AMPK (p-AMPK; clone 40H9) were purchased from Cell Signaling Technology (Danvers, Mass). IHC staining of LKB1 and pAMPK in FFPE samples was scored by a board-certified pathologist (JME) based on a previously published 4-category scoring system (0-3) for LKB1 and p-AMPK applied to FFPE samples of lung cancer. ${ }^{16}$ In the scoring scale, 0 represents no cytoplasmic or membranous staining; 1 , weak staining; 2 , moderate staining; and 3 , strong staining.

\section{Publicly Available Esophageal Cancer Datasets}

LKB1 copy number, expression, and protein expression data were obtained from The Cancer Genome Atlas (TCGA) esophageal carcinoma (provisional) dataset. This dataset contains samples and methodologies from the TCGA data for esophagus and stomach cancers previously published by the TCGA Research Network, Analysis Working Group. ${ }^{17}$ The esophageal carcinoma dataset (provisional) contains 186 esophageal 
tumors from 185 patients, 89 of which with an esophageal adenocarcinoma histology. LKB1 copy number was determined by GISTIC2 analysis, ${ }^{18}$ whereas $L K B 1$ mRNA expression was determined by RNA sequencing (RNA-seq, V2 RSEM). The $q$ value in GISTIC2 is the $P$ value adjusted for false discovery rate. Protein expression of LKB1 and p-AMPK was determined by reverse-phase protein assay (RPPA). The RNA-seq and RPPA methods have been described previously. ${ }^{19}$ Statistical calculations (Student's $t$ test for normal distributions, and the Mann-Whitney test for non-normal distributions) were performed in Prism 7 (GraphPad Software, La Jolla, Calif), with a $P$ value $<.05$ considered statistically significant. The normality of the data was determined by the Shapiro-Wilk normality test. One sample was censored from the diploid LKB1 sample set with regard to age at diagnosis because it failed the outlier test (Grubb's test).

The Oncomine database (www.oncomine.org) was used to analyze the mRNA expression analysis of $L K B 11^{20}$ The Kim esophageal dataset was used, in which gene expression was determined by the Illumina Human6 v2.0 Expression Beadchip array as described previously. ${ }^{21}$ Expression of $L K B 1$ in nontumor esophageal samples $(\mathrm{n}=28)$, BEs $(\mathrm{n}=15)$, and EACs $(n=75)$ is reported as $\log 2$ median-centered intensity, with differences between histological groups determined using Student's $t$ test in Oncomine. A $P$ value $<.05$ was considered statistically significant.

\section{Cell Lines and siRNA Knockdown}

SK-GT-4 human EAC cells were obtained from the European Collection of Authenticated Cell Cultures/Sigma-Aldrich (St Louis, Mo). These cells were maintained in RPMI-1640, with $10 \%$ fetal bovine serum and pen/strep (Thermo Fisher Scientific, Waltham, Mass). Cell lines were maintained at $37^{\circ} \mathrm{C}$ in $5 \% \mathrm{CO}_{2}$. Small-interfering RNA (siRNA) was used to suppress LKB1 expression in SK-GT-4 cells. siRNAs targeting LKB1 were purchased from QIAGEN (Germantown, Md). Transient transfection was performed by plating cells in 96-well plates. Cells were allowed to adhere overnight and were then transfected with luciferase siRNA (control) or functionally verified LKB1 siRNAs (LKB1 siRNAs 5 and 6) targeted to the human $L K B 1$ transcript at a final concentration of $150 \mathrm{nmol} / \mathrm{L}$. Transfection was carried out using RNAiMax transfection reagent (Thermo Fisher Scientific) according to the manufacturer's instructions. Cell lysates were collected at 48 hours post-transfection for immunoblot analysis.

\section{Immunoblot Analysis}

Immunoblot analyses were performed following standard procedures for adherent cell lines. Cells were washed twice with $1 \times$ phosphate buffered saline and then lysed in RIPA lysis buffer $(0.1 \mathrm{M}$ phenylmethylsulfonyl fluoride, $\mathrm{NaOV}$, and ultra-pure distilled $\mathrm{H}_{2} \mathrm{O}$ ) and $1 \times$ HALT Protease Inhibitor Cocktail from $100 \times$ (Thermo Fisher Scientific). Scraped cells were transferred to prechilled 1.5-mL Eppendorf tubes. The collected cells were then sonicated and pelleted by centrifugation, after which the supernatant was transferred to a new 1.5-mL Eppendorf tube. Protein concentration was determined by the BCA assay (Bio-Rad Laboratories, Hercules, Calif), and total protein lysates were separated by sodium dodecyl sulfatepolyacrylamide gel electrophoresis and transferred to nitrocellulose membranes. Blots were blocked with $5 \%$ milk in Tris-buffered saline/0.1\% Tween 20 (TBST). Primary antibodies were diluted in 5\% milk in TBST and incubated overnight at $4{ }^{\circ} \mathrm{C}$ on a shaker. Blots were developed using Amersham ECL Prime Western Blotting Detection Reagent (GE Healthcare, Marlborough, Mass) and visualized using the LI-COR Odyssey Fc imaging system (LI-COR Biosciences, Lincoln, Neb). Antibodies to LKB1, p-AMPK, and actin were obtained from Cell Signaling Technology (Danvers, Mass). Actin served as a loading control.

\section{Cell Growth Assay}

Cell growth was determined using the CyQUANT Cell Proliferation Assay (Thermo Fisher Scientific, Waltham, Mass), according to the manufacturer's protocol for adherent cell lines. Cells were split into 96well assay plates for siRNA transfection as described previously and maintained at $37^{\circ} \mathrm{C} / 5 \% \mathrm{CO}_{2}$ for 72 hours post-transfection. Assay plates were read after the addition of $1 \times$ CyQUANT dye-binding solution and a 60 minute incubation at $37^{\circ} \mathrm{C}$. The fluorescence intensity of each sample was read using an EnVision 2102 multilabel plate reader (PerkinElmer, Waltham, Mass), with excitation at $\sim 485 \mathrm{~nm}$ and emission detection at $\sim 530 \mathrm{~nm}$. Statistical significance, defined at $P<.05$, was determined by analysis of variance with Dunnett's multiple comparisons test to determine differences from controls using GraphPad Prism 7. Normality of the data was determined using the Shapiro-Wilk normality test.

\section{RESULTS}

\section{LKB1 Protein Expression in EAC}

In this study, we constructed an exploratory esophageal TMA consisting of 14 gastroesophageal adenocarcinomas (EACs, $\mathrm{n}=8$; gastric adenocarcinomas, $\mathrm{n}=2$; GE junction adenocarcinomas, $\mathrm{n}=3$ ), 1 esophageal squamous cell carcinoma, 7 high-grade dysplasias with evidence of carcinoma, 1 intestinal metaplasia, and normal stomach tissue and small bowel control tissue. The protein expression of LKB1 and p-AMPK were determined by IHC. LKB1 functions as the primary kinase responsible for phosphorylating and activating the AMPK. ${ }^{22}$ Table 1 shows that EAC tumors, as well as gastric adenocarcinomas and GE junction adenocarcinomas, are likely to have low or no (IHC score 0-1) LKB1 protein expression. Normal tissues (stomach and small bowel) demonstrated elevated LKB1protein levels. LKB1 protein expression was also significantly correlated $(P<.05)$ with $\mathrm{p}$-AMPK protein staining in this TMA, as determined by Pearson's correlation coefficient, consistent with IHC staining in lung cancer. ${ }^{16}$ Figure 1 shows a representative EAC tumor with no LKB1 protein staining but with LKB1 expression in cells of the surrounding stroma. Thus, these observations suggest that a subset of EAC tumors has lost protein expression of LKB1.

\section{LKB1 Copy Number and mRNA Expression}

To further characterize and validate the loss of LKB1 in EACs, we mined publicly available expression datasets. The TCGA esophageal carcinoma (provisional) dataset contains 186 samples from 185 patients, including 89 EAC specimens. In the full dataset, a statistically significant ( $q=0.0000086)$ deletion of $19 \mathrm{p} 13.3$ was determined by GISTIC2.0, ${ }^{18}$ which includes the $L K B 1$ locus. When analyzing only the EAC samples, we found that $58 \%$ harbored a shallow deletion of $L K B 1$ as determined by GISTIC2.0, whereas $37.5 \%$ were diploid for $L K B 1$ (Table 2). Figure 2, $A$ and $B$ shows that compared to diploid EAC tumors, EAC tumors harboring LKB1 deletions had statistically significant reductions in both mRNA and protein expression of LKB1. In those EAC tumors with loss of $L K B 1$, the protein expression of p-AMPK was also significantly reduced compared with diploid EAC tumors (Figure 2,C). 
TABLE 1. LKB1 and p-AMPK protein expression in human esophageal tumors

\begin{tabular}{|c|c|c|c|c|c|c|}
\hline Patient & Histology & $\begin{array}{l}\text { Average LKB1 } \\
\text { IHC score }\end{array}$ & $\begin{array}{c}\text { Average p-AMPK } \\
\text { IHC score }\end{array}$ & Tumor punches & Sex & $\begin{array}{c}\text { Age at } \\
\text { diagnosis, yr }\end{array}$ \\
\hline 1 & EAC & 0.25 & 0 & 4 & Male & 74 \\
\hline 2 & EAC & 0 & 0 & 2 & Male & 57 \\
\hline 3 & EAC & 0.5 & 1.5 & 2 & Male & 73 \\
\hline 4 & EAC & 0.5 & 1.5 & 2 & Male & 51 \\
\hline 5 & EAC & 0.5 & 0 & 2 & Male & 67 \\
\hline 6 & EAC & 0.5 & 1 & 2 & Male & 69 \\
\hline 7 & EAC & No core & No core & 1 & Male & 78 \\
\hline 8 & EAC & 1 & 1 & 2 & Male & 72 \\
\hline 9 & ESCC & 0.5 & 0 & 2 & Female & 71 \\
\hline 10 & Gastric adenocarcinoma & 0 & 0 & 1 & Female & 78 \\
\hline 11 & Gastric adenocarcinoma & 0.5 & 1 & 2 & Male & 60 \\
\hline 12 & GE junction adenocarcinoma & 0 & 1 & 2 & Female & 68 \\
\hline 13 & GE junction adenocarcinoma & 0 & 0.5 & 2 & Male & 69 \\
\hline 14 & GE junction adenocarcinoma & 0.5 & 1 & 2 & Female & 67 \\
\hline 15 & High-grade dysplasia & 1 & 1 & 2 & Male & 49 \\
\hline 16 & High-grade dysplasia/carcinoma & 0.75 & 0.75 & 4 & Male & 70 \\
\hline 17 & High-grade dysplasia/carcinoma & 0 & 0.5 & 3 & Male & 46 \\
\hline 18 & High-grade dysplasia/carcinoma & 1 & 1 & 4 & Male & 80 \\
\hline 19 & High-grade dysplasia/carcinoma & 0.333333 & 2.333333 & 3 & Male & 67 \\
\hline 20 & High-grade dysplasia/carcinoma & 1 & 1 & 2 & Male & 68 \\
\hline 21 & High-grade dysplasia/carcinoma & 1 & 2 & 2 & Male & 53 \\
\hline 22 & Intestinal metaplasia & 1 & 2.333333 & 4 & Male & 51 \\
\hline 23 & Mucinous adenocarcinoma & 1 & 1 & 2 & Male & 68 \\
\hline 24 & Normal stomach & 2 & 1.5 & 2 & Male & 64 \\
\hline 25 & Small bowel control & 3 & 2.5 & 2 & NA & NA \\
\hline
\end{tabular}

Immunohistochemistry protein scores for LKB1 and p-AMPK were determined by a board-certified pathologist across the TMA. Scores represent the average score per number of punches from the tumor. Clinical variables are included per patient. $L K B 1$, Liver kinase B1; IHC, immunohistochemistry; $p$-AMPK, phosphorylated-protein kinase AMPactivated catalytic subunit alpha 1;EAC, esophageal adenocarcinoma; $E S C C$, esophageal squamous cell carcinoma; $G E$, gastroesophageal; $N A$, not available.

To further validate the loss of $L K B 1$ expression in EAC, we analyzed the Kim esophagus dataset in Oncomine (www.oncomine.org). ${ }^{21}$ Figure 2, $D$ shows significantly lower LKB1 mRNA expression in EAC tumors $(\mathrm{n}=75)$ compared with normal esophageal tissue $(\mathrm{n}=28)$ and $\mathrm{BE}$ $(\mathrm{n}=15)$. To determine whether loss of $L K B 1$ was associated with clinical variables, we compared the EAC tumors with shallow deletion against diploid tumors with respect to age at diagnosis. As shown in Figure 3, age at diagnosis was significantly earlier in the patients with $L K B 1$ deletion compared with those with diploid tumors (median, 62 years vs 74 years; $P<.05$ ). $L K B 1$ deletion was not significantly correlated with overall survival.

\section{LKB1 Suppression and EAC Cell Growth}

To better understand the role of LKB1 loss on EAC cell growth, we suppressed LKB1 expression via functionally verified siRNA (siLKB1-6 and siLKB1-6) in SK-GT-4 cells.
Figure 4, A demonstrates suppression of LKB1 protein expression in the siLKB1-treated cells compared with control (siRNA against luciferase). As a functional validation, the protein expression of p-AMPK was also shown to be suppressed in the siLKB1-treated cells compared with control. In Figure 4, $B$, suppression of LKB1 using multiple siRNAs targeting LKB1 resulted in significantly $(P<.05)$ enhanced cell growth at 72 hours compared with siRNA targeting luciferase (control).

\section{DISCUSSION}

In the present study, we have demonstrated that loss of LKB1 expression is a common occurrence in esophageal adenocarcinoma. Tumors in patients with EAC showed loss of protein staining of LKB1. Shallow deletion of $L K B 1$ DNA in EAC occurs in $58 \%$ of tumors and is correlated with significant reductions in LKB1 mRNA and protein expression. Validating our findings, LKBI mRNA 


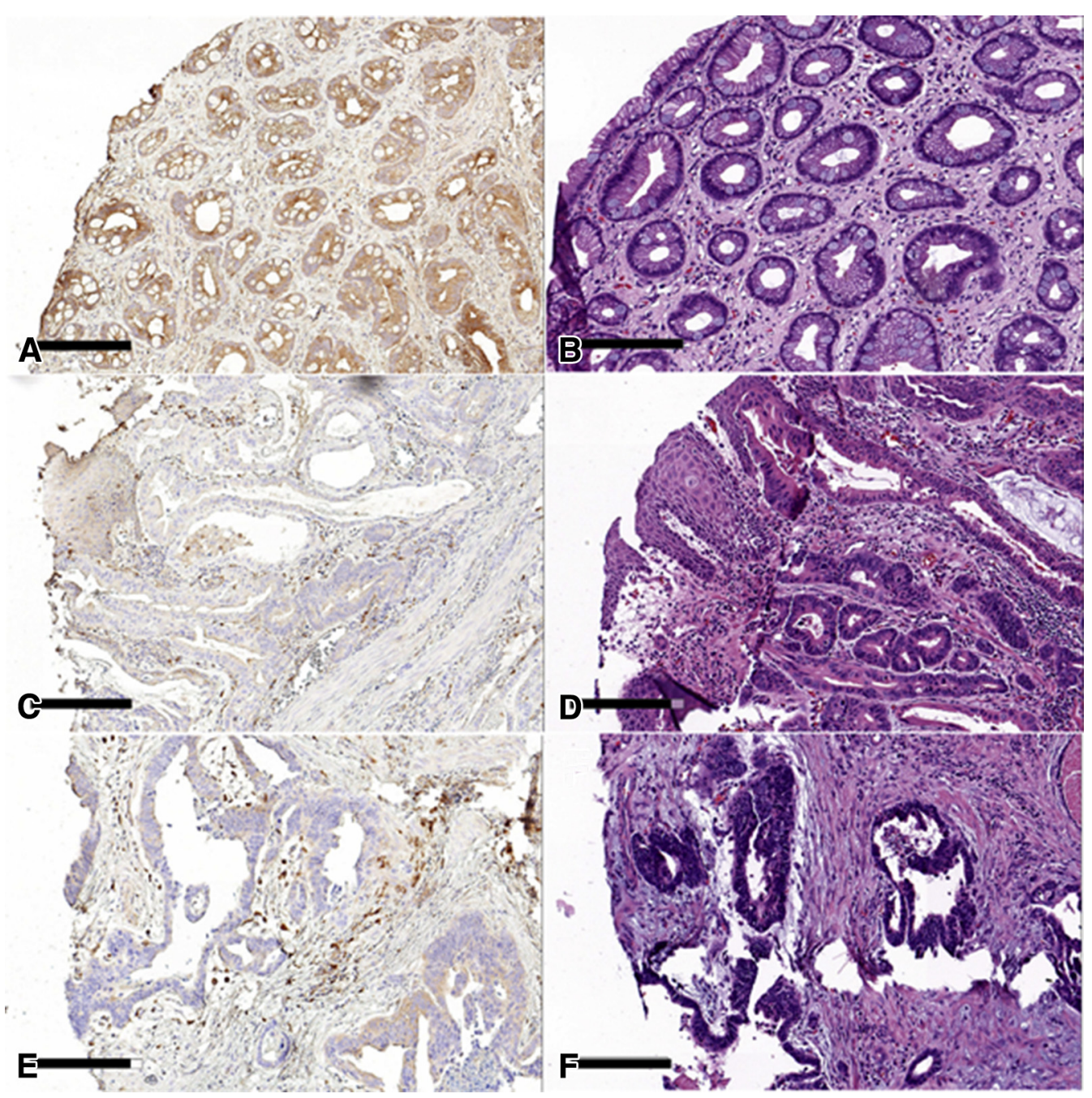

FIGURE 1. Representative images from the esophageal adenocarcinoma (EAC) tissue microarray. Immunohistochemistry staining of liver kinase B1 (left) with hematoxylin and eosin staining of serial sections (right) in nondysplastic Barrett's (BE) (A and B), dysplastic BE (C and D), and EAC (E and F). (Scale bar: $200 \mu$.)

expression was significantly reduced in another set of EAC tumors compared with nontumor esophageal tissue and BE. LKBI loss was associated with earlier age at diagnosis of EAC. We further demonstrated that loss of LKB1 via siRNA enhanced EAC cell growth in vitro. Collectively, our data suggest that loss of LKB1 may contribute to EAC tumorigenesis.

TABLE 2. LKB1 copy number alterations in the TCGA esophageal carcinoma provisional dataset

\begin{tabular}{lcccc}
\hline Cancer type & $\begin{array}{c}\text { Deep } \\
\text { deletion, } \mathbf{n}\end{array}$ & $\begin{array}{c}\text { Shallow } \\
\text { deletion, } \mathbf{n}\end{array}$ & Diploid, $\mathbf{n}$ & Gain, $\mathbf{n}$ \\
\hline EAC & 0 & 51 & 33 & 4 \\
ESCC & 2 & 43 & 40 & 11 \\
\hline
\end{tabular}

LKBI putative copy number, as analyzed in GISTIC2, were determined in EAC and ESCC specimens. EAC, Esophageal adenocarcinoma; ESCC, esophageal squamous cell carcinoma.
LKB1 was initially discovered as a causal, genomic alteration in the hereditary cancer syndrome PJS. ${ }^{7}$ Of interest, patients with PJS are known to be susceptible to gastrointestinal tumors, including those of the esophagus. ${ }^{11}$ However, little is known about the prevalence and role of LKB1 expression in EAC tumorigenesis and progression. Our data suggest that LKB1 protein levels are lost in a subset of EAC patient tumors. These data support findings from $\mathrm{Gu}$ and colleagues, ${ }^{12}$ who reported that esophageal squamous cell carcinoma cell lines had reduced LKB1 protein levels, and that a small number of EA samples showed reduced LKB1 protein expression compared with matched normal tissue. ${ }^{12}$ Using publicly available datasets, we found that shallow deletion of $L K B 1$ is a common genomic aberration in EAC $(58 \%)$, and that $L K B 1 \mathrm{mRNA}$ levels are reduced in EAC compared with in nontumor esophageal tissue and BE. Unlike in lung adenocarcinomas, in which 

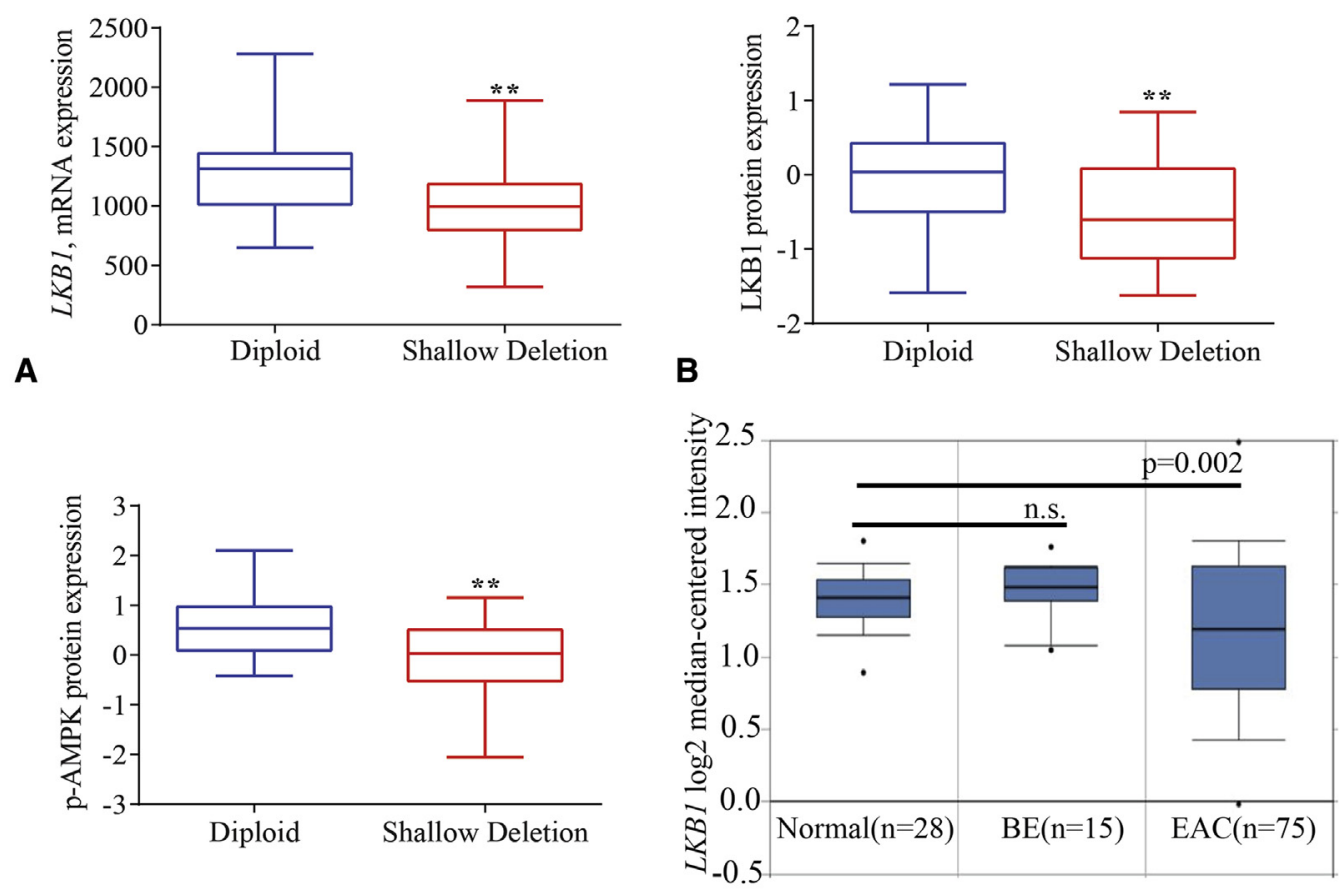

C

D

FIGURE 2. Shallow deletion of liver kinase B1 ( $L K B 1)$ in esophageal adenocarcinoma $(E A C)$ tumors correlates with reduced $L K B 1 \mathrm{mRNA}$, protein levels, and activated AMPK. A-C, Using The Cancer Genome Atlas esophageal carcinoma (provisional) dataset, $L K B 1 \mathrm{mRNA}$ (diploid, $\mathrm{n}=33$; shallow deletion, $\mathrm{n}=51)$ as determined by RNA sequencing and LKB1 (diploid, $\mathrm{n}=22$; shallow deletion, $\mathrm{n}=21)$ and phosphorylated AMPK $(p-A M P K)($ diploid, $\mathrm{n}=22$; shallow deletion, $\mathrm{n}=22$ ) as determined by RPPA were examined in EAC tumors with shallow deletion of $L K B 1$ compared with diploid tumors as determined by GISTIC2 analysis. **P<.05. The box extends from the 25 th to the 75 th percentile, with the whiskers extending from minimum to maximum values. $\mathrm{D}$, Using the Kim esophagus dataset in Oncomine, LKB1 mRNA levels were determined in normal (non-tumor esophageal tissue), BE, and EAC. A $P$ value $<.05$ was considered statistically significant. $B E$, Barrett's esophagus.

point mutations in $L K B 1$ are prevalent, leading to loss of expression and function, ${ }^{8}$ point mutations in LKB1 appear rare in EAC. Loss of $L K B 1$ in other tumor types is governed by genomic deletion or methylation. ${ }^{10}$ As seen in other solid tumor types, loss of the tumor suppressor LKB1 is a common event in EAC, suggesting a role in tumorigenesis and progression.

The progression from $\mathrm{BE}$ to EAC is thought to progress though increasing grades of dysplasia before the development of adenocarcinoma. ${ }^{23,24}$ As such, there is intense interest in understanding the point during this BE to EAC progression at which molecular changes occur. Mutations in TP53, the most common genomic aberration in EAC, ${ }^{17}$ have been detected as early as nondysplastic BE. ${ }^{5}$ Inactivation of $C D K N 2 A$ also has been observed at the earliest stages of the transition from BE to EAC, ${ }^{25}$ whereas oncogenic receptor amplifications are considered later events. ${ }^{5}$ In this study, reduced protein expression was observed in patient tumors harboring high-grade dysplasia, albeit in a small number of samples. In the Kim esophagus dataset in Oncomine, LKB1 mRNA was significantly reduced in EAC compared with $\mathrm{BE}$, again in a small number of $\mathrm{BE}$ samples. Thus, there is a need for further investigation of the role of LKB1 loss in the transition from BE to EAC, and when it occurs, which could have prognostic and therapeutic implications.

In a meta-analysis of solid tumors, the loss of $L K B 1$ was correlated with reduced overall survival, increased tumor stage, and tumor metastasis. ${ }^{26}$ In our data, the loss of $L K B 1$ is associated with disease onset at an earlier age, although we acknowledge the possible role of lead time in such a measure. We further found that suppression of LKB 1 protein by siRNA significantly enhanced EAC tumor cell growth in vitro compared with controls. This is consistent with data for other esophageal histological subtypes. In TE10 ESCC cells, the overexpression of $L K B 1$ was found to inhibit cell growth, whereas the suppression of $L K B 1$ by shRNA enhanced cell growth. ${ }^{13}$ In TE1 cells, another ESCC cell line, the overexpression of $L K B 1$ suppressed cell growth in vitro via suppression of STAT3 signaling. ${ }^{14}$ Across a number of solid tumor types, in both cell lines and animal models, loss of $L K B 1$ expression or activity results in more aggressive tumors. In non-small cell lung carcinoma animal models, the deletion of $L \mathrm{kbl}$ in the context of mutant Kras was shown to be sufficient to induce distant metastases and shorter survival time. ${ }^{27}$ Thus, the loss of LKB1 in EAC may promote more aggressive tumor types; however, 


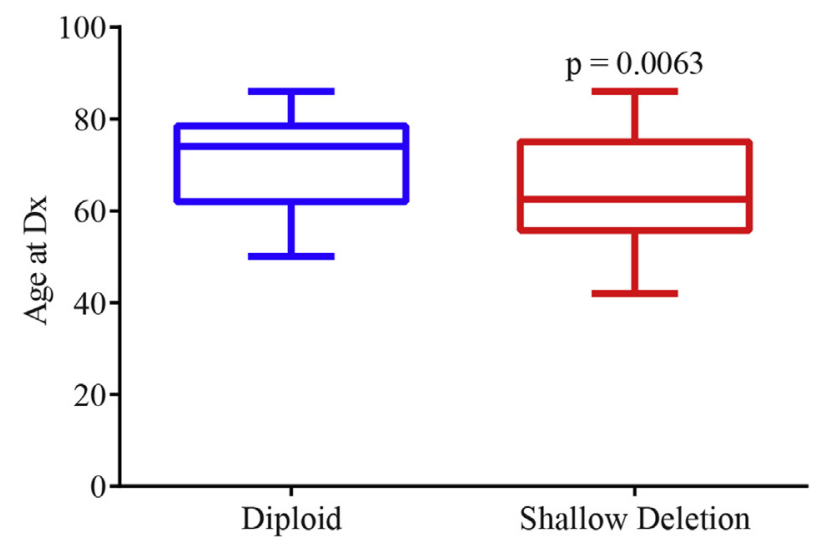

FIGURE 3. Loss of liver kinase B1 (LKB1) in esophageal adenocarcinoma (EAC) correlates with earlier age at diagnosis. Using The Cancer Genome Atlas esophageal carcinoma (provisional) dataset, age at patient diagnosis $(D x)$ was examined in EAC tumors with shallow deletion of LKB1 $(\mathrm{n}=50)$ compared with diploid tumors $(\mathrm{n}=32)$ as determined by GISTIC2 analysis. A $P$ value $<.05$ was considered statistically significant. The box extends from the 25 th to the 75 th percentile, with the whiskers extending from minimum to maximum values.

further studies are needed to investigate this hypothesis in robust human datasets.

A growing number of therapeutic strategies have demonstrated preclinical efficacy in the molecular context of $L K B 1$ loss. The involvement of LKB1 in the regulation of cellular metabolism through AMPK prompted studies using metformin or phenformin to suppress tumor cells deficient for $L K B 1 .^{28,29}$ Our laboratory has shown that lung tumor cells deficient for $L K B 1$ are preferentially susceptible to energetic stress by 2-deoxyglucose exposure. ${ }^{15,30}$ Because LKB1 has been shown to modulate mammalian target of rapamycin (mTOR) signaling, mTOR inhibitors have shown preclinical promise in treating LKB1-deficient tumor cells. ${ }^{31}$ In our study, EAC tumors with loss of $L K B 1$ were more likely to have elevated expression of mTOR signaling components (mTOR and TSC2) than EAC tumors with diploid $L K B 1$ (data not shown). More recently, tumor cells with loss of $L K B 1$ were shown to be more sensitive to poly (ADP ribose) polymerase inhibition. ${ }^{32}$ Because our data suggest that LKB1 protein levels are lost in EAC, therapeutic strategies that have shown promise in other settings of LKB1 deficiency may be promising in EAC as well. Future research will be aimed at exploring effective therapeutic strategies for those EAC tumors that have lost LKB1 expression.

We recognize some limitations associated with this study, including the small number of samples included in the protein analysis by IHC. We also recognize that serial samples from the same patient would allow more definitive studies as to when $L K B 1$ is lost during disease progression. Furthermore, data from multiple sites can have differences in previous treatments and time to analysis, although the results
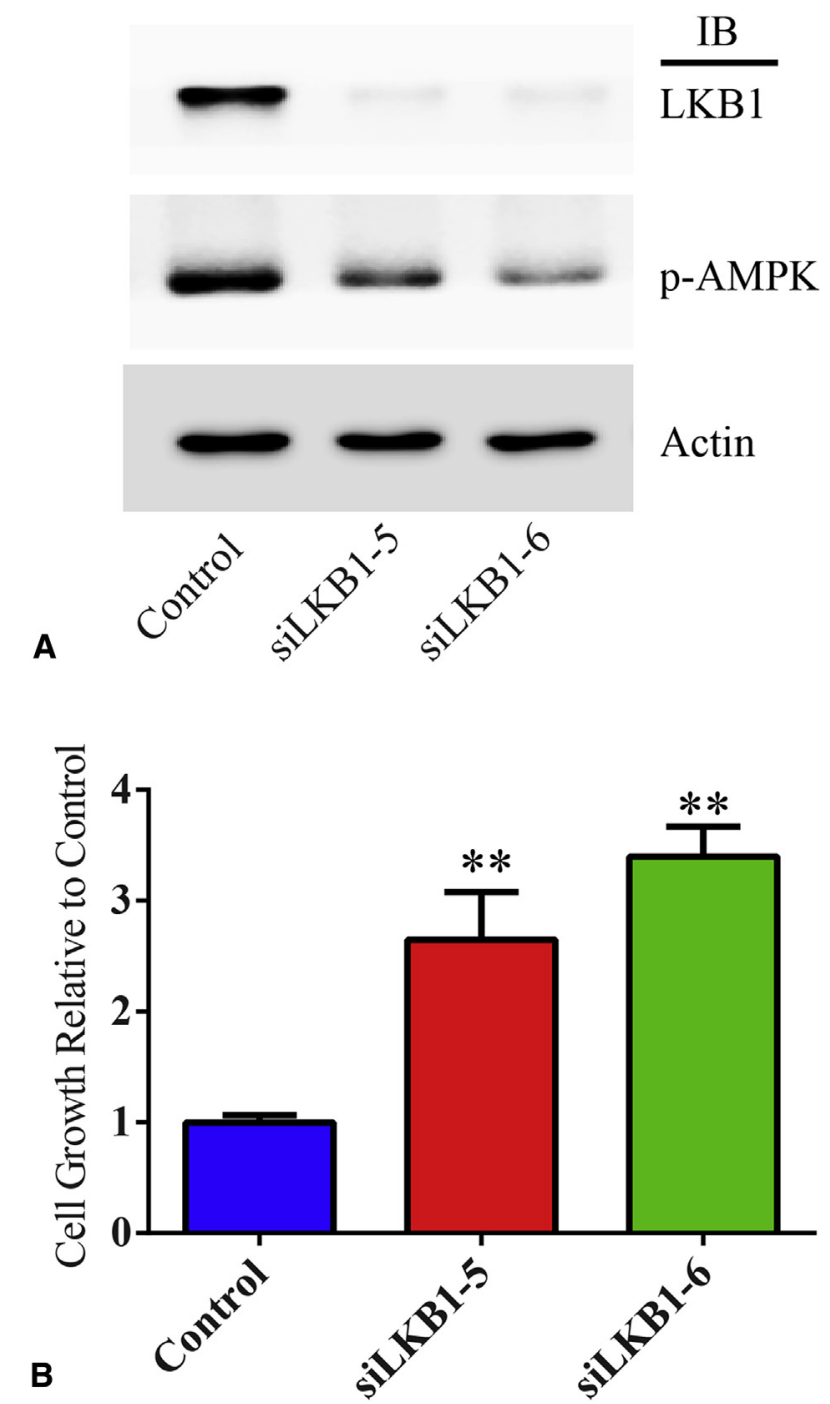

FIGURE 4. Suppression of liver kinase B1 ( $L K B 1)$ enhances esophageal adenocarcinoma (EAC) cell growth in vitro. A, LKB1 was suppressed in SK-GT-4 EAC cells by multiple siRNAs, with a luciferase-targeting siRNA serving as a control. Protein lysates were separated by sodium dodecyl sulfate-polyacrylamide gel electrophoresis and immunoblotted for indicated antibodies at 48 hours after siRNA exposure, with actin used as a loading control. B, Cell growth was assessed at 72 hours after siRNA transfection by the CyQUANT Cell Proliferation Assay. $* * P<.05$. $I B$, Immunoblot; $p$-AMPK, phosphorylated-protein kinase AMP-activated catalytic subunit alpha 1 .

across the institutions were validated in this study. Finally, we recognize that time to diagnosis can be affected by multiple clinical variables, including method of initial discovery. Although most of the patients in the TCGA dataset were symptomatic, a few were identified on screening. As such, we have focused on the association, but currently are developing larger, better-annotated datasets to more thoroughly explore the outcomes associated with loss of LKB1 expression. 


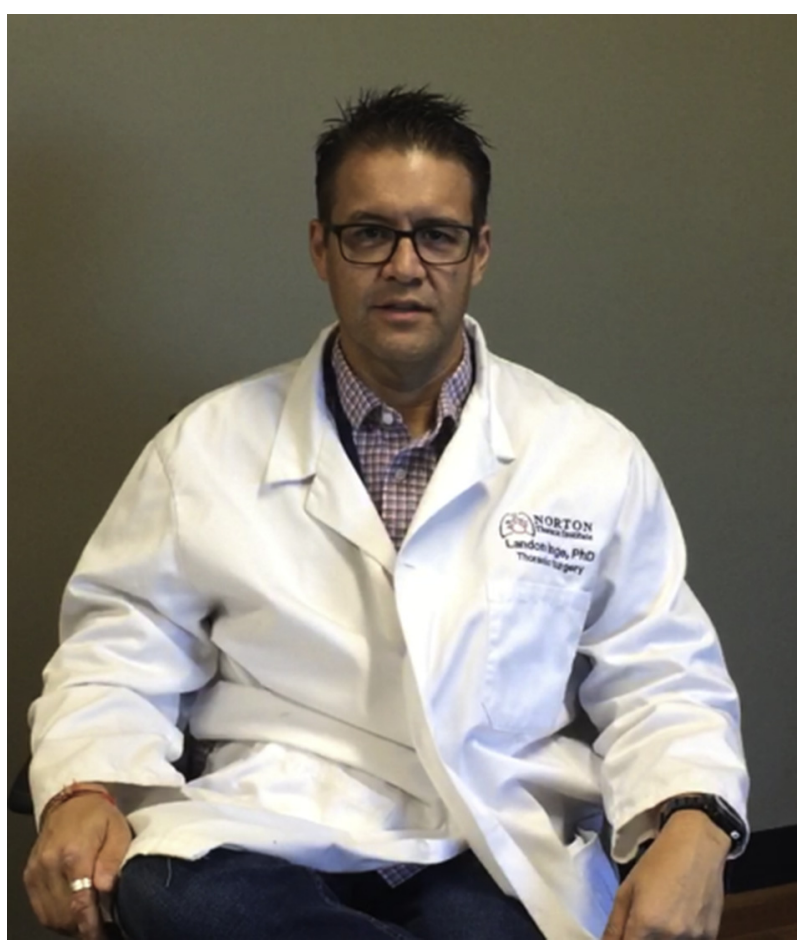

VIDEO 1. The senior author, Dr Landon Inge, describing the significance of these findings with regard to a better understanding of the progression and potential treatment of esophageal adenocarcinoma. Video available at: http://www.jtcvsonline.org/article/S0022-5223(17)32760-5/fulltext.

EAC remains a deadly disease with a dismal 5-year survival rate. Associations with obesity and gastric reflux, 2 conditions on the rise in the United States and worldwide, suggest that EAC will continue to be prevalent and without effective treatment. A deeper understanding of the molecular events that drive EAC should uncover rational therapeutic strategies. These data suggest that the tumor suppressor $L K B 1$ is lost in a subset of EAC tumors. Because the loss of $L K B 1$ leads to poor prognosis and tumor progression and has therapeutic implications, this molecular alteration in EAC warrants further exploration (Video 1).

\section{Conflict of Interest Statement}

Authors have nothing to disclose with regard to commercial support.

The authors thank Tim Fey and Sreeja Biswas Roy for assembling the clinical variables used in the EAC TMA.

\section{References}

1. Hur C, Miller M, Kong CY, Dowling EC, Nattinger KJ, Dunn M, et al. Trends in esophageal adenocarcinoma incidence and mortality. Cancer. 2013;119:1149-58.

2. Siegel RL, Miller KD, Jemal A. Cancer statistics, 2017. CA Cancer J Clin. 2017; 67:7-30.

3. Abbas G, Krasna M. Overview of esophageal cancer. Ann Cardiothorac Surg. 2017;6:131-6.
4. Dulak AM, Stojanov P, Peng S, Lawrence MS, Fox C, Stewart C, et al. Exome and whole-genome sequencing of esophageal adenocarcinoma identifies recurrent driver events and mutational complexity. Nat Genet. 2013;45:478-86.

5. Stachler MD, Taylor-Weiner A, Peng S, McKenna A, Agoston AT, Odze RD, et al. Paired exome analysis of Barrett's esophagus and adenocarcinoma. Nat Genet. 2015;47:1047-55

6. Secrier M, Li X, de Silva N, Eldridge MD, Contino G, Bornschein J, et al. Mutational signatures in esophageal adenocarcinoma define etiologically distinct subgroups with therapeutic relevance. Nat Genet. 2016;48:1131-41.

7. Hemminki A, Markie D, Tomlinson I, Avizienyte E, Roth S, Loukola A, et al. A serine/threonine kinase gene defective in Peutz-Jeghers syndrome. Nature. 1998; 391:184-7.

8. Sanchez-Cespedes M, Parrella P, Esteller M, Nomoto S, Trink B, Engles JM, et al. Inactivation of LKB1/STK11 is a common event in adenocarcinomas of the lung. Cancer Res. 2002;62:3659-62.

9. Shackelford DB, Shaw RJ. The LKB1-AMPK pathway: metabolism and growth control in tumour suppression. Nat Rev Cancer. 2009;9:563-75.

10. Lee SM, Choi JE, Na YK, Lee EJ, Lee WK, Choi YY, et al. Genetic and epigenetic alterations of the LKB1 gene and their associations with mutations in TP53 and EGFR pathway genes in Korean non-small cell lung cancers. Lung Cancer. 2013;81:194-9.

11. Bartosova Z, Zavodna K, Krivulcik T, Usak J, Mlkva I, Kruzliak T, et al. STK11/ LKB1 germline mutations in the first Peutz-Jeghers syndrome patients identified in Slovakia. Neoplasma. 2007;54:101-7.

12. Gu Y, Lin S, Li JL, Nakagawa H, Chen Z, Jin B, et al. Altered LKB1/CREB regulated transcription co-activator (CRTC) signaling axis promotes esophageal cancer cell migration and invasion. Oncogene. 2012;31:469-79.

13. Liu K, Luo Y, Tian H, Yu KZ, He JX, Shen WY. The tumor suppressor LKB1 antagonizes WNT signaling pathway through modulating GSK3beta activity in cell growth of esophageal carcinoma. Tumour Biol. 2014;35: 995-1002.

14. Wang YQ, Dai WM, Chu XY, Yang B, Zhao M, Sun Y. Downregulation of LKB1 suppresses Stat3 activity to promote the proliferation of esophageal carcinoma cells. Mol Med Rep. 2014;9:2400-4.

15. Inge LJ, Friel JM, Richer AL, Fowler AJ, Whitsett T, Smith MA, et al. LKB1 inactivation sensitizes non-small cell lung cancer to pharmacological aggravation of ER stress. Cancer Lett. 2014;352:187-95.

16. Nakada Y, Stewart TG, Peña CG, Zhang S, Zhao N, Bardeesy N, et al. The LKB1 tumor suppressor as a biomarker in mouse and human tissues. PLoS One. 2013;8: e73449.

17. Cancer Genome Atlas Research Network, Analysis Working Group, Asan University, BC Cancer Agency, Brigham and Women's Hospital, Broad Institute, et al. Integrated genomic characterization of oesophageal carcinoma. Nature. 2017;541:169-75.

18. Mermel CH, Schumacher SE, Hill B, Meyerson ML, Beroukhim R, Getz G. GISTIC2.0 facilitates sensitive and confident localization of the targets of focal somatic copy-number alteration in human cancers. Genome Biol. 2011;12:R41.

19. Cancer Genome Atlas Research Network. Comprehensive molecular characterization of gastric adenocarcinoma. Nature. 2014;513:202-9.

20. Rhodes DR, Yu J, Shanker K, Deshpande N, Varambally R, Ghosh D, et al. ONCOMINE: a cancer microarray database and integrated data-mining platform. Neoplasia. 2004;6:1-6.

21. Kim SM, Park YY, Park ES, Cho JY, Izzo JG, Zhang D, et al. Prognostic biomarkers for esophageal adenocarcinoma identified by analysis of tumor transcriptome. PLoS One. 2010;5:e15074.

22. Hong SP, Leiper FC, Woods A, Carling D, Carlson M. Activation of yeast Snf1 and mammalian AMP-activated protein kinase by upstream kinases. Proc Natl Acad Sci U S A. 2003;100:8839-43.

23. Cameron AJ, Souto EO, Smyrk TC. Small adenocarcinomas of the esophagogastric junction: association with intestinal metaplasia and dysplasia. Am J Gastroenterol. 2002;97:1375-80.

24. Stein HJ, Siewert JR. Barrett's esophagus: pathogenesis, epidemiology, functional abnormalities, malignant degeneration, and surgical management Dysphagia. 1993;8:276-88.

25. Klump B, Hsieh CJ, Holzmann K, Gregor M, Porschen R. Hypermethylation of the CDKN2/p16 promoter during neoplastic progression in Barrett's esophagus. Gastroenterology. 1998;115:1381-6.

26. Xiao J, Zou Y, Chen X, Gao Y, Xie M, Lu X, et al. The prognostic value of decreased LKB1 in solid tumors: a meta-analysis. PLoS One. 2016;11: $\mathrm{e} 0152674$. 
27. Ji H, Ramsey MR, Hayes DN, Fan C, McNamara K, Kozlowski P, et al. LKB1 modulates lung cancer differentiation and metastasis. Nature. 2007;448:807-10.

28. Algire C, Amrein L, Bazile M, David S, Zakikhani M, Pollak M. Diet and tumor LKB1 expression interact to determine sensitivity to anti-neoplastic effects of metformin in vivo. Oncogene. 2011;30:1174-82.

29. Shackelford DB, Abt E, Gerken L, Vasquez DS, Seki A, Leblanc M, et al. LKB1 inactivation dictates therapeutic response of non-small cell lung cancer to the metabolism drug phenformin. Cancer Cell. 2013;23:143-58.

30. Inge LJ, Coon KD, Smith MA, Bremner RM. Expression of LKB1 tumor suppressor in non-small cell lung cancer determines sensitivity to 2-deoxyglucose. J Thorac Cardiovasc Surg. 2009;137:580-6.
31. Mahoney CL, Choudhury B, Davies H, Edkins S, Greenman C, Haaften GV, et al LKB1/KRAS mutant lung cancers constitute a genetic subset of NSCLC with increased sensitivity to MAPK and mTOR signalling inhibition. $\mathrm{Br} J$ Cancer. 2009; 100:370-5

32. Wang YS, Chen J, Cui F, Wang H, Wang S, Hang W, et al. LKB1 is a DNA damage response protein that regulates cellular sensitivity to PARP inhibitors. Oncotarget. 2016;7:73389-401.

Key Words: esophageal adenocarcinoma, liver kinase B1 (LKB1), Barrett's esophagus

Readers who found these articles interesting may also like to read the following papers found in recent and future issues of our sister publications, Seminars in Thoracic and Cardiovascular Surgery and Operative Techniques in Thoracic and Cardiovascular Surgery!

Thoracic: Esophageal Cancer

STATE OF THE ART: Quality of Life after Open or Minimally Invasive Esophagectomy in Patients With Esophageal Cancer-A Systematic Review. Emanuela Taioli. Semin Thoracic Surg 2017: 377-390.

ORIGINAL SUBMISSION: Esophagectomy in Patients with HIV/AIDS: a Viable Option. Michael Mwachiro. Semin Thoracic Surg 2017: In press.

ORIGINAL SUBMISSION: Minimally Invasive Esophagectomy Provides Equivalent Survival to Open Esophagectomy: An Analysis of the National Cancer Data Base. Brian Mitzman. Semin Thoracic Surg 2017: 244-253.

Editorial Commentary: Is the Glass Half Full or Empty? Minimally Invasive Esophagectomy in an Age of Alternative Facts. Benjamin Wei. Semin Thoracic Surg 2017: 254-255.

ORIGINAL SUBMISSION: Node-Negative Esophageal Cancer With Short-Interval Isolated Metastasis to the Gallbladder: A Case Report. Christopher M. Hart. Semin Thoracic Surg 2017: 115-117.

Editorial Commentary: Signet Rings Around the World. Philip Carrott. Semin Thoracic Surg 2017: 118.

ORIGINAL SUBMISSION: Total Laryngopharyngoesophagectomy and Tracheal Resection With Free Tissue-Reinforced Gastric Pull-Up. Michael Reilly. Oper Tech Thorac Cardiovasc 2016: 31-46.

ORIGINAL SUBMISSION: Mediastinal Tracheostomy With Vessel Transposition and Minimally Invasive Transhiatal Esophagectomy. Christa N. Grant. Oper Tech Thorac Cardiovasc 2016: 47-64. 